Bundesgesundheitsbl -

Gesundheitsforsch - Gesundheitsschutz

2002 · 45:323-331 @ Springer-Verlag 2002

Originalien und Übersichtsarbeiten

C.Meyer · S.Reiter · A.Siedler · W.Hellenbrand · G.Rasch

Robert Koch-Institut, Berlin

\title{
Über die Bedeutung von Schutzimpfungen
}

\section{Epidemiologie, Durchimpfungsraten, Programme}

beschriebenen Defiziten in Deutschland ergeben sich die Anforderungen an zukünftige Gesundheitsziele und an die Ausgestaltung von Impfprogrammen zur Förderung des Impfgedankens.

\section{Schlüsselwörter}

Impfung $\cdot$ Durchimpfung $\cdot$ Impfprogramme Akzeptanz

Stellenwert und Wirkungsgrad. Grundlage der Weiterentwicklung von Impfprogrammen müssen kontinuierlich erhobene, valide und belastbare Daten zu Erkrankungshäufigkeiten, Seroprävalenz, Durchimpfungsraten und Impfkomplikationen sowie die Evaluation bestehender Programme sein. Durch das seit 1.1.2001 geltende Infektionsschutzgesetz (IfSG) werden bestehende Datenlücken in der Erhebung der Häufigkeiten impfpräventabler Erkrankungen und der Umsetzung von Impfprogrammen geschlossen. Der dargestellte Rückgang der Erkrankungshäufigkeiten im Zusammenhang mit der Einführung von Impfungen und mit dem Anstieg von Durchimpfungsraten belegt ihre Wirksamkeit sowie die Effektivität von Impfprogrammen. Die Akzeptanz von Impfungen wird von vielen unterschiedlichen Faktoren beeinflusst, die es bei der Ausgestaltung von Impfprogrammen zu berücksichtigen gilt. Maßnahmen mit nachgewiesener Verbesserung der Durchimpfungsraten beziehen sich anhand internationaler Studien auf Impfregister, automatisierte Erinnerungs- und Recallsysteme, Aufklärungsmaßnahmen für Bevölkerung und Fachöffentlichkeit, Kostenerstattung, Abbau von Zugangsbarrieren und Qualitätssicherungsmaßnahmen. Aus den urch Schutzimpfungen wird sowohl ein individueller Krankheitsschutz als auch ein Populationsschutz (Herdimmunität) möglich. Die Eradikation der Pocken, die anstehende Elimination der Poliomyelitis (voraussichtliche Zertifizierung 2003) und die geplante Elimination der Masern (voraussichtliche Zertifizierung 2010) zeigen eindrücklich die Bedeutung von Schutzimpfungen. Der Erfolg und die Akzeptanz von Impfmaßnahmen hängen von einer Reihe sehr unterschiedlicher Faktoren ab (z. B. Inzidenz der Erkrankung, Schwere der Erkrankung, Häufigkeit von Krankheitskomplikationen, Effektivität des Impfstoffes, Nebenwirkung der Impfung, Erreichbarkeit der Zielpopulation, Inanspruchnahme, Akzeptanz der Impfung in der Bevölkerung, Kostenerstattung, institutionelle Verankerung des Impfwesens, gesundheitspolitische Unterstützung u.a.). Bei der Planung und Durchführung von Impfprogrammen sind die- se unterschiedlichen Gesichtspunkte ausreichend zu berücksichtigen.

Grundlage der Ausgestaltung von Impfprogrammen müssen kontinuierlich erhobene, valide und belastbare $\mathrm{Da}$ ten zu Erkrankungshäufigkeiten, Seroprävalenz, Durchimpfungsraten, Impfkomplikationen sowie zur Evaluation bestehender Programme sein.

Basierend auf der epidemiologischen Bedeutung einer Erkrankung und einer günstigen Risiko-Nutzen-Abwägung empfiehlt die Ständige Impfkommission (STIKO) bestimmte Schutzimpfungen entweder für die gesamte Bevölkerung oder nur für besonders gefährdete Personengruppen. Die Kosten für allgemein empfohlene Impfungen werden in der Regel von den Krankenkassen oder dem Arbeitgeber übernommen. Empfehlungen der STIKO werden von den Landesgesundheitsverwaltungen in landesweite Empfehlungen im Sinne von Rechtsverordnungen übernommen. Diese landesweiten Empfehlungen sind auch Grundlage der gesetzlich garantierten Entschädigungsregeln bei Auftreten eines Impfschadens. Hintergrund für Programme, die die Entschädigung im Falle eines Impfschadens regeln, ist die Sicherung des Einzelnen bei gleichzeitig bestehendem Interesse der Allgemeinheit zur Durchführung von Impfungen.

Dr.Christiane Meyer

Robert Koch-Institut, Nordufer 20,13353 Berlin E-Mail: MeyerC@rki.de 
Bundesgesundheitsbl -

Gesundheitsforsch - Gesundheitsschutz

2002 • 45:323-331 ๑) Springer-Verlag 2002

C.Meyer · S.Reiter · A.Siedler •

W.Hellenbrand·G.Rasch

\section{The Importance of Vaccination}

\section{Epidemiology, Vaccination Programs, Immunisation Rates}

\section{Abstract}

Vaccination, along with the improvement of social and hygienic living conditions, has played a key role in the prevention and control of infectious diseases due to its effectiveness, its favourable risk-benefit ratio and strong acceptance on the part of society and health policy makers. The further development of vaccination programs depends on the continual collection of valid data on the incidence of vaccine preventable diseases, immunisation rates, seroprevalence, vaccine related complications as well as on the evaluation of existing programs. The Protection against Infection Act (IfSG) in effect since January 1,2001 has closed important gaps in the surveillance of vaccine preventable diseases and the implementation of vaccination programs in Germany. The data presented here show the decrease in the incidence of vaccine preventable diseases following implementation of vaccination and increasing vaccine coverage in Germany, underlining the effectiveness of vaccines as well as vaccination programs. The acceptance of vaccination is influenced by various factors that should be taken into account in the organization of vaccination programs. Measures shown to improve vaccination rates in international studies include the establishment of vaccine registries, reminder and recall systems, education of the public as well as health care workers, reimbursement of providers, improvement of access to providers and quality control measures. The deficits in Germany described here reveal further requirements for the organization of vaccination programs, for the improvement of awareness for the importance of vaccine coverage and for the achievement of health related goals.

\section{Keywords}

Vaccination · Immunisation Rates · Vaccination Programs · Acceptance

\section{Originalien und Übersichtsarbeiten}

\section{Erfassungssysteme und Daten- grundlagen zu impfpräventablen Erkrankungen}

Durch das seit dem 1.1.2001 geltende Infektionsschutzgesetz (IfSG) werden bestehende Datenlücken in der Erhebung impfpräventabler Erkrankungen und der Analyse der Umsetzung von Impfprogrammen geschlossen [1]. Die zusätzliche Einführung der Meldepflicht für den Verdacht einer Masernerkrankung, für den Erregernachweis bei Haemophilus-influenzae-Typ-b-Infektionen und der Frühsommermeningoenzephalitis (FSME) ist ein wichtiger Schritt für die notwendige Evaluation bestehender Impfstrategien zur Eindämmung dieser Erkrankungen. Eine Übersicht über die Meldepflicht von Erkrankungen und Erregern mit empfohlenen allgemeinen Schutzimpfungen findet sich in der Tabelle 1.

Daten aus Routinestatistiken und passiver Surveillance unterliegen jedoch methodischen Einschränkungen (z.B. Untererfassung, fehlende Differenzierung, fehlende Stratifizierung und Adjustierung u.a.), so dass ergänzende Daten aus aktiver Surveillance, aus Sentinels und aus alternativen Erfassungssystemen unbedingt notwendig sind. Für einen Teil der impfpräventablen Erkrankungen stehen Daten aus solchen Systemen und Sentinels zur Verfügung. Aber auch hier sind methodische Einschränkungen, insbesondere im Hinblick auf die Verallgemeinerung für das gesamte Bundesgebiet, zu berücksichtigen. In den neuen Bundesländern (NBL) liegen z. B. aufgrund der Meldepflicht die in der DDR bestanden hat, Daten für Masern, Röteln, Mumps und Pertussis vor, die für die alten Bundesländer (ABL) fehlen. Für die Darstellung der Masernmorbidität bietet zudem das seit 1999 durchgeführte Sentinel der Arbeitsgemeinschaft Masern (AGM) eine gute zusätzliche Datengrundlage. Aus dem Vergleich der Daten aus Meldepflicht und Sentinel lassen sich wichtige Erkenntnisse sowohl für die epidemiologische Situation als auch für die Funktionalität der beiden Systeme gewinnen $[2,3]$.

Die Erhebung von Daten zu impfpräventablen Erkrankungen dient in diesem Sinne nicht vordringlich der epidemiologischen Grundlagenwissenschaft, sondern soll relevante und prioritäre Probleme der öffentlichen Gesundheit im $\mathrm{Zu}$ sammenhang mit Infektionskrankheiten aufzeigen und Grundlage zielorientierter effektiver Handlungsmaßnahmen sein.
Tabelle 1

Übersicht über vorhandene Meldedaten impfpräventabler Erkrankungen, für die allgemeine Impfempfehlungen bestehen

\begin{tabular}{|c|c|c|c|c|}
\hline $\begin{array}{l}\text { Impfpräventable } \\
\text { Erkrankung }\end{array}$ & $\begin{array}{l}\text { Meldepflicht } \\
\text { der Erkrankung } \\
\text { gemäß IfSG }\end{array}$ & $\begin{array}{l}\text { Meldepflicht für } \\
\text { den Erregernachweis } \\
\text { gemäß IfSG }\end{array}$ & $\begin{array}{l}\text { Nationales } \\
\text { Referenzzentrum }\end{array}$ & $\begin{array}{l}\text { Sonstige } \\
\text { Erhebungen }\end{array}$ \\
\hline Diphtherie & $x$ & $x$ & - & - \\
\hline Tetanus & $-^{*}$ & - & - & - \\
\hline Polio & $x$ & $x$ & $x$ & $\begin{array}{l}\text { AFP- } \\
\text { Surveillance }\end{array}$ \\
\hline Pertussis & - & - & - & $\begin{array}{l}\text { Neue } \\
\text { Bundesländer }\end{array}$ \\
\hline Hepatitis B & $x$ & $x$ & - & - \\
\hline $\begin{array}{l}\text { Hämophilus } \\
\text { influenzae }\end{array}$ & - & $x$ (seit 2001) & - & $\begin{array}{l}\text { ESPED u. } \\
\text { Labor-ESPED }\end{array}$ \\
\hline Masern & $x$ (seit 2001) & $x($ seit 2001) & $x$ & Sentinel \\
\hline Mumps & - & - & $\mathrm{x}$ & $\begin{array}{l}\text { Neue } \\
\text { Bundesländer }\end{array}$ \\
\hline Röteln & $\begin{array}{l}\text { Nur konnatale } \\
\text { Infektion }\end{array}$ & - & $x$ & $\begin{array}{l}\text { Neue } \\
\text { Bundesländer }\end{array}$ \\
\hline
\end{tabular}

* Meldepflicht 2001 aufgehoben

ESPED Erhebungseinheit für seltene pädiatrische Erkrankungen in Deutschland, AFP Acute flaccid paralysis (akute schlaffe Parese). 
Tabelle 2

Gemeldete absolute Erkrankungszahlen (RKI) einiger impfpräventabler Erkrankungen

\begin{tabular}{|c|c|c|c|c|c|}
\hline & 1980 & 1985 & 1990 & 1995 & 2000 \\
\hline $\begin{array}{l}\text { Masernfälle* } \\
\text { (DDR bzw.NBL) }\end{array}$ & 28.745 & 568 & 190 & 80 & 73 \\
\hline $\begin{array}{l}\text { Mumpserkrankungen* } \\
\text { (DDR bzw.NBL) }\end{array}$ & 126.886 & 44.397 & 9647 & 354 & 268 \\
\hline $\begin{array}{l}\text { Röteln* } \\
\text { (DDR bzw.NBL) }\end{array}$ & $\begin{array}{l}22830 \\
(1983)\end{array}$ & 69.230 & 22.269 & 507 & 352 \\
\hline Hepatitis B & Ca. 6000 & 7088 & 4608 & 6146 & 4542 \\
\hline $\begin{array}{l}\text { Pertussis* } \\
\text { (DDR bzw.NBL) }\end{array}$ & 258 & 306 & 57 & 369 & 2845 \\
\hline Diphtherie & 19 & 4 & 6 & 4 & 0 \\
\hline Tetanus & 21 & 18 & 18 & 11 & 8 \\
\hline Polio & 8 & 4 & 3 & 3 (VAAP) & 1 (VAAP) \\
\hline Meningokokken & 1301 & 1269 & 877 & 651 & 757 \\
\hline
\end{tabular}

*Daten aus den neuen Bundesländern (RKI).

\section{Häufigkeit impfpräventabler Erkrankungen}

In den letzten 20 Jahren ist die Häufigkeit, mit der impfpräventable Erkrankungen auftreten, rückläufig (Tabelle 2). Dieser Trend zeigt sich eindrucksvoll auch am diesbezüglichen Rückgang der Sterbefälle, wobei in diesem Zusammenhang immer auch die verbesserten medizinischen Leistungen zu berücksichtigen sind (Tabelle 3 ).

\section{Masern - Mumps - Röteln}

In den alten Bundesländern konnte die Zahl der Masern-, Mumps- und Rötelnerkrankungen bis einschließlich 2000

\begin{tabular}{lrrr}
$\begin{array}{l}\text { Tabelle } 3 \\
\text { Sterbefälle durch einige impfpräven- } \\
\text { table Erkrankungen (Statistisches }\end{array}$ \\
Bundesamt, Todesursachenstatistik) \\
& 1980 & 1990 & 1998 \\
\hline & 23 & 1 & 2 \\
\hline Masern & & 4 & 3 \\
Mumps & 0 & 1 & 0 \\
Röteln & 105 & 132 & 158 \\
$\begin{array}{l}\text { Hepatitis B } \\
\text { (akut) }\end{array}$ & & & \\
$\begin{array}{l}\text { Pertussis } \\
\text { Diphtherie }\end{array}$ & 2 & 8 & 0 \\
Tetanus & 12 & 8 & 4 \\
Meningokokken & 114 & 89 & 68
\end{tabular}

einzig über Modellrechnungen auf Basis von Daten der Hospitalstatistik geschätzt werden. Mit dem seit 1999 implementierten Masernsentinel und der seit 2001 bestehenden Meldepflicht für Masern stehen nun aber auch für diese Bundesländer Daten aus jüngerer Zeit zur Verfügung. Abbildung 1 zeigt die Hospitalisierungsraten für Masern in den Bundesländern im Vergleich. Während der letz- ten epidemischen Welle 1996 wurden in den alten Bundesländern 6,5 von100.000 Einwohner wegen Masernerkrankungen hospitalisiert, in den neuen Bundesländern mit der deutlich besseren Durchimpfungsrate dagegen nur 1,7 von 100.000 Einwohnern. Insgesamt ist ein Rückgang an Krankenhausbehandlungen wegen Masern zu beobachten. Allerdings liegt die Zahl der Krankenhausbehandlungen in den alten Bundesländern auch aktuell noch etwa doppelt so hoch wie in den neuen Bundesländern (1999: ABL: 0,8/100.000, NBL: 0,3/100.000). Die Zahl der Masernerkrankungen in den 9oer-Jahren weitgehend stabil niedrig. Ausbrüche (1996) haben aber auch hier aufgrund der nach der Wiedervereinigung zeitweilig schlechteren Durchimpfungsraten $\mathrm{zu}$ einem vorübergehenden Inzidenzanstieg geführt (1996: NBL: 3,7/100.000; 2000: NBL 0,5/100.000, 2001 NBL: 0,7/100.000, s. Abb. 1).

Auch die Ergebnisse des Masernsentinels $[2,3,4]$ verdeutlichen, dass die Masernmorbidität regional auf sehr unterschiedlichem Niveau liegt. Die Auswertung der IfSG-Meldedaten des Jahres 2001 bestätigt diese Beobachtungen. Sie zeigt gemeldete mittlere Inzidenzen von fünf bis zehn Erkrankungen pro 100.000 Einwohnern mit regionalen Spitzen von neuen Bundesländern blieb auch in den

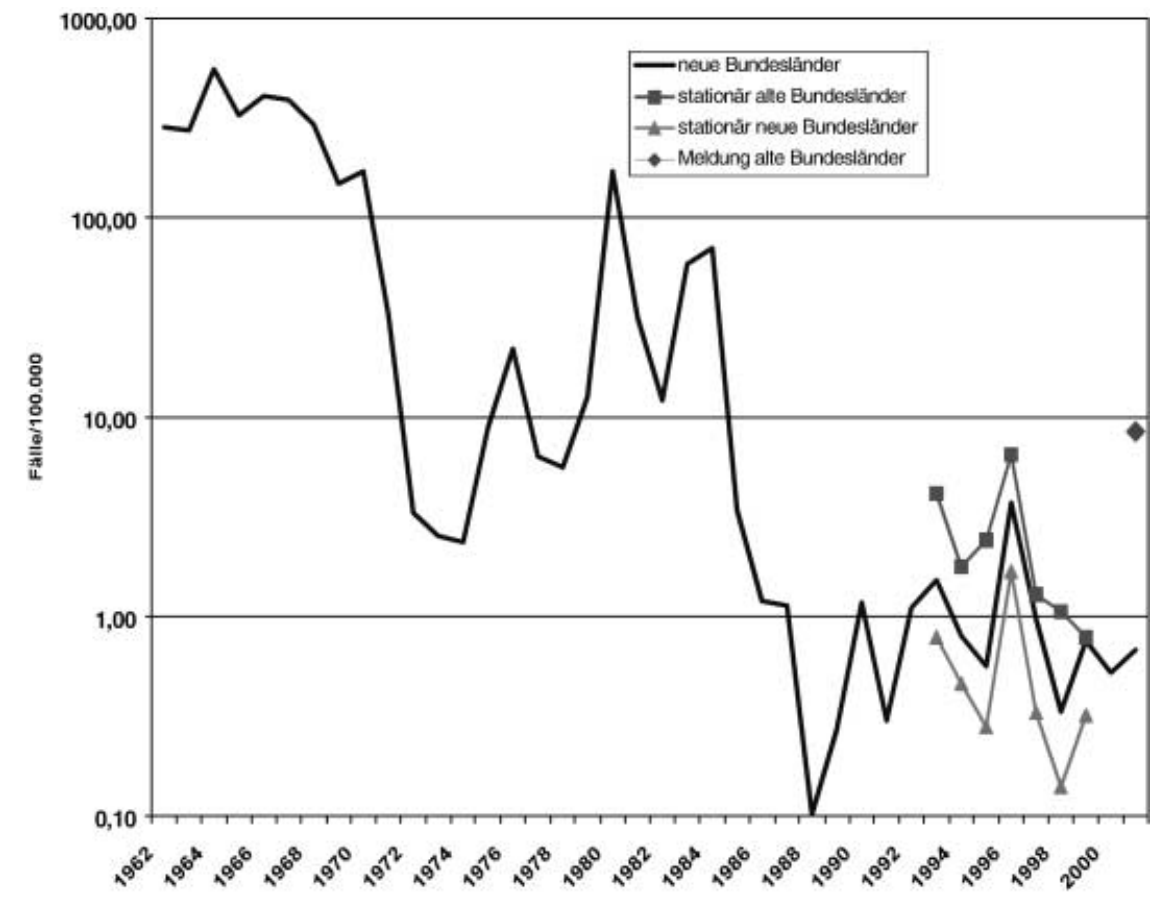

Abb. $1 \Delta$ Maserninzidenz und stationäre Behandlungsfälle in der DDR und in den neuen Bundesländern 


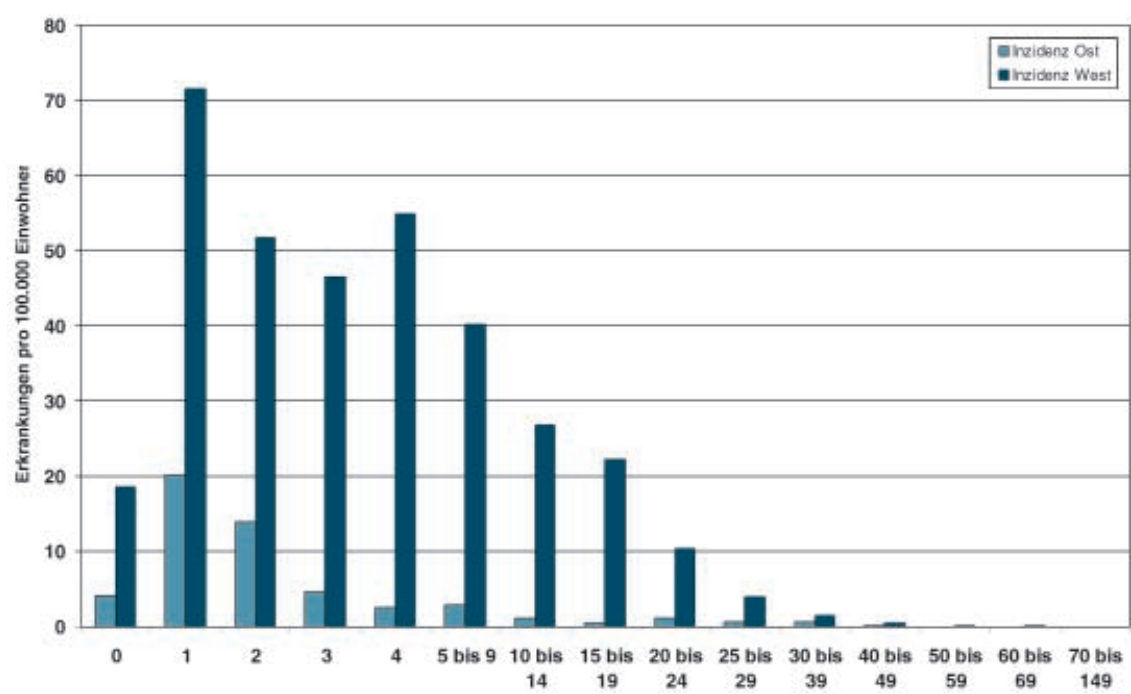

Abb. 2 A Altersverteilung der nach IfSG gemeldeten Masernfälle 2001

weit über 20 und niedrigen Inzidenzen von unter einer Erkrankung pro 100.000 Einwohnern. Die Altersverteilung der im Jahr 2001 gemeldeten Erkrankungsfälle macht deutlich, dass ein nicht unbedeutender Anteil des Erkrankungsgeschehens durch Ausbrüche in Schulen und Gemeinschaftseinrichtungen zu erklären ist (Abb. 2). $\mathrm{Zu}$ berücksichtigen ist hier jedoch, dass trotz der absoluten Meldezahl von über 6.00o Erkrankungen im Jahr 2001 methodische Probleme wie Untererfassung, Konsultationswahrscheinlichkeit, Diagnosewahrscheinlichkeit, unterschiedliche Meldebereitschaft und unterschiedliche Zugangswege zum Gesundheitswesen eine Rolle spielen.
Die Meldedaten spiegeln derzeit das Erkrankungsgeschehen also nur unvollständig wider. Auch die labordiagnostische Klärung eines Erkrankungsfalles (unabdingbare Voraussetzung im Rahmen eines effektiven Eliminationsprogrammes) erfolgt bisher nur in rund $40 \%$ der Fälle [3].

Daten zu Mumps- und Rötelnerkrankungen liegen für ganz Deutschland nur aus der Hospitalstatistik vor (Abb.3, 4). Inzidenzen für die neuen Bundesländer sind der in diesen Ländern fortgeführten Meldepflicht zu entnehmen. Dabei ist zu berücksichtigen, dass gegen beide Erkrankungen in der ehemaligen DDR nicht geimpft wurde. Nach Einfüh-

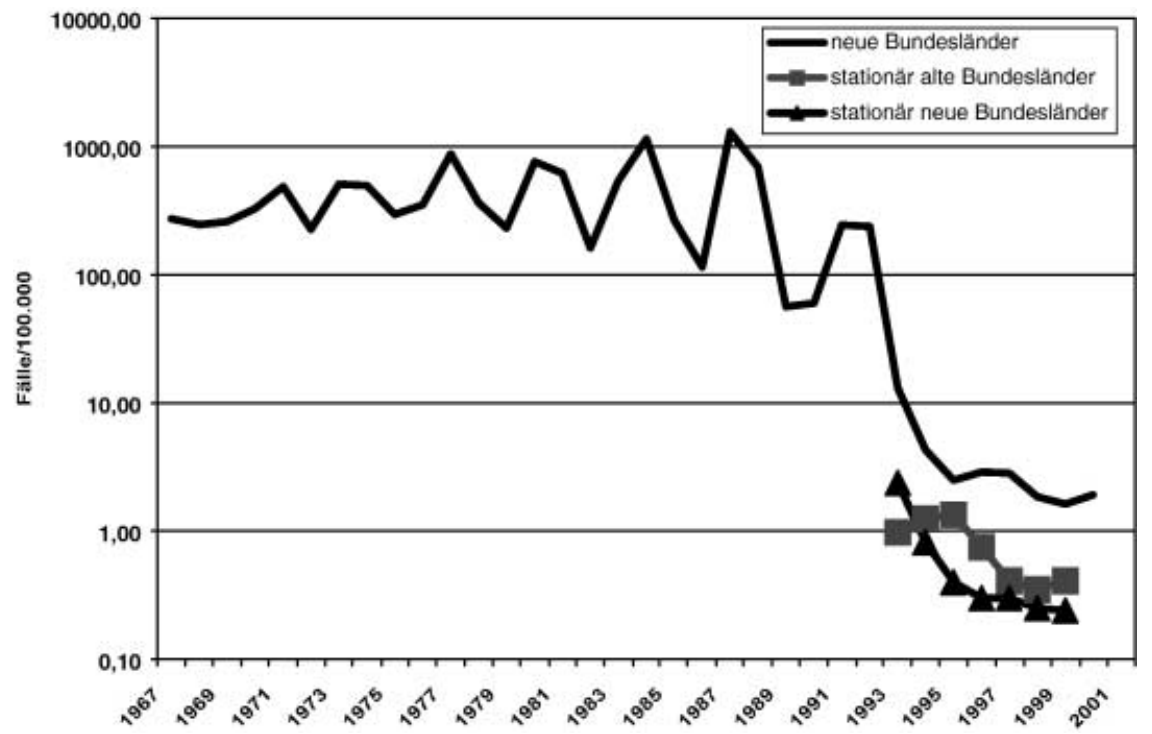

Abb. $3 \Delta$ Mumpsinzidenz und stationäre Behandlungsfälle in der DDR und in den neuen Bundesländern rung des Kombinationsimpfstoffs im Jahr 1990 und steigenden Impfraten zeigt sich in den neuen Bundesländern ein erfreulicher Rückgang sowohl der Inzidenz- als auch der Hospitalisierungsraten. Die Zahlen demonstrieren eindrücklich die Bedeutung der Schutzimpfung für die Eindämmung von Mumpsund Rötelnerkrankungen (Röteln: 1989: 560/100.000, 2000: 2,52/100.000 (Spannweite 0,3-4,3/100.000); Mumps 1989: 56,3/100.000; 2000: 1,92/100.000 (Spannweite $0,449 / 100.000-2,646 / 100.000)$ ).

\section{Pertussis}

Aufgrund der unterschiedlichen Impfstrategien in der DDR und in der Bundesrepublik differieren die Zahlen von Pertussiserkrankungen in den alten und neuen Bundesländern. Bestand in der DDR seit 1974 eine generelle Impfpflicht gegen Pertussis, wurde in den alten Bundesländern erst 1995 nach Zulassung des azellulären Pertussisimpfstoffs und der erneuten Impfempfehlung der STIKO (1991) wieder eine relevante Durchimpfungsrate erreicht (Tabelle 4 und Abb. 5, 6).

Für die neuen Bundesländer zeigt Abb. 5 den Inzidenzverlauf der Pertussiserkrankungen von 1977 bis 2000. Nachdem in den 7oer- und 8oer-Jahren eine durchschnittliche Inzidenz von 1-2 Fällen pro 100.000 Einwohnern beobachtet wurde, stieg diese von $0,2 / 100.000 \mathrm{im}$ Jahr 1991 über 8,6/100.00o im Jahr 1997 auf 20,4/100.000 im Jahr 2000 an. Ursächlich hierfür sind lokale Ausbrüche und epidemische Häufungen in einzelnen Bundesländern (Brandenburg 58,4/ 100.0002000 , vgl. [5]). Aus der Hospitalstatistik ergibt sich sowohl für die neuen als auch für die alten Bundesländer ein erfreulicher Rückgang der Hospitalisierungsraten (1993: ABL: 4/100.000, NBL: 1,51/100.000; 1999: ABL: 1,71/100.000, NBL: 0,79/100.000, vgl.Abb. 1, 2). Wurden 1993 noch 2.890 Kinder (vorwiegend unter fünf Jahren) wegen einer Pertussiserkrankung stationär behandelt, so waren es 1999 nur 1.218. In den neuen Bundesländern erkranken vor allem Jugendliche. Nach Erhebungen in der Erhebungseinheit für seltene pädiatrische Erkrankungen in Deutschland (ESPED) sind $13 \%$ der hospitalisierten Kinder in den alten Bundesländern, aber $45 \%$ der hospitalisierten Kinder in den neuen Bundesländern zwischen sechs und 15 Jahre alt [6]. Diese epidemiologische Entwicklung und 
Tabelle 4

Durchimpfungsraten und Spannweiten in Prozent der vorgelegten Impfausweise bei Schuleintritt in den Jahren von 1999 bis 2001 ( $n=623.279$ aus 14 Bundesländern)

\begin{tabular}{|c|c|c|c|c|c|c|}
\hline & Gesamt & $\begin{array}{l}\text { Spannweite } \\
\text { (Range) }\end{array}$ & $\begin{array}{l}\text { Alte } \\
\text { Bundesländer }\end{array}$ & $\begin{array}{l}\text { Spannweite } \\
\text { (Range) }\end{array}$ & $\begin{array}{l}\text { Neue } \\
\text { Bundesländer }\end{array}$ & $\begin{array}{l}\text { Spannweite } \\
\text { (Range) }\end{array}$ \\
\hline Diphtherie & $96,9 \%$ & $92,9 \%-98,5 \%$ & $97,0 \%$ & $92,9 \%-98,5 \%$ & $95,20 \%$ & $93,1 \%-98,5 \%$ \\
\hline Tetanus & $97,0 \%$ & $93,0 \%-98,6 \%$ & $97,20 \%$ & $93,0 \%-98,6 \%$ & $95,30 \%$ & $93,0 \%-98,6 \%$ \\
\hline Pertussis & $73,8 \%$ & $53,3 \%-91,6 \%$ & $71,90 \%$ & $53,3 \%-90,2 \%$ & $88,60 \%$ & $87,5 \%-91,6 \%$ \\
\hline Hib & $81,3 \%$ & $74,9 \%-90,9 \%$ & $80,90 \%$ & $74,9 \%-89,8 \%$ & $84,90 \%$ & $75,3 \%-90,9 \%$ \\
\hline IPV & $95,5 \%$ & $94,0 \%-97,3 \%$ & $95,50 \%$ & $94,0 \%-97,3 \%$ & $96,0 \%$ & $94,4 \%-96,9 \%$ \\
\hline Hepatitis B & $37,2 \%$ & $9.9 \%-61,8 \%$ & $39,0 \%$ & $9,9 \%-59,9 \%$ & $27,00 \%$ & $11,0 \%-61,8 \%$ \\
\hline Masern & $90,2 \%$ & $86,2 \%-96,2 \%$ & $89,70 \%$ & $86,2 \%-93,5 \%$ & $94,20 \%$ & $92,4 \%-96,2 \%$ \\
\hline Mumps & $89,8 \%$ & $83,0 \%-95,9 \%$ & $89,30 \%$ & $83,0 \%-93,4 \%$ & $93,70 \%$ & $91,8 \%-95,9 \%$ \\
\hline Röteln & $83,3 \%$ & $54,0 \%-95,1 \%$ & $82,10 \%$ & $54,0 \%-90,3 \%$ & $93,10 \%$ & $91,4 \%-95,1 \%$ \\
\hline
\end{tabular}

das Wissen um den fehlenden lebenslangen Schutz sowohl nach Impfung als auch nach Erkrankung hat die STIKO im Jahr 2000 dazu veranlasst, die Auffrischungsimpfung für Jugendliche bzw. das Nachholen der Grundimmunisierung (zu jedem Zeitpunkt im Kindes- und Jugendalter) zu empfehlen. Probleme bei der Kostenübernahme durch die Krankenkassen mögen eine Rolle bei der bisher noch nicht befriedigenden Umsetzung dieser Empfehlung spielen.

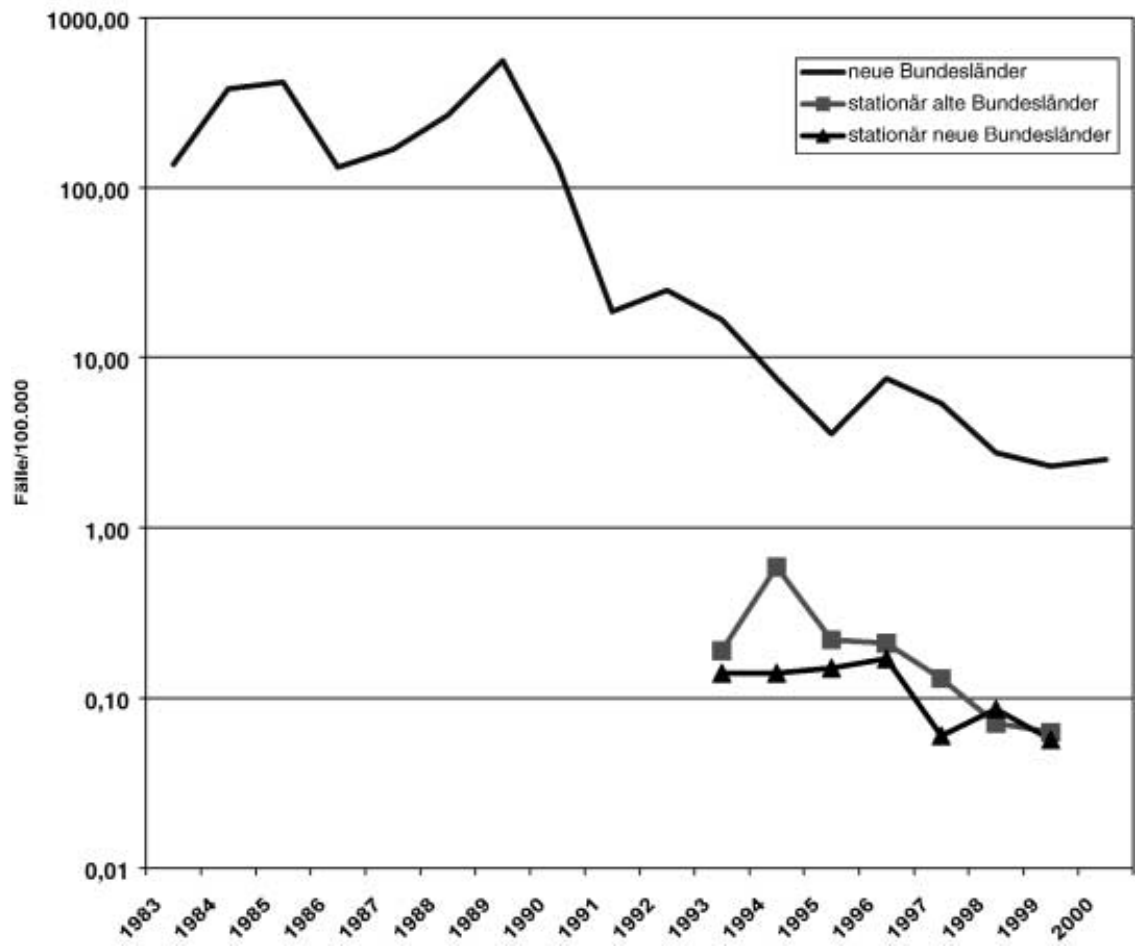

Abb. $4 \Delta$ Rötelninzidenz und stationäre Behandlungsfälle in der DDR und in den neuen Bundesländern

\section{Poliomyelitis}

Seit 1962 - in der DDR seit 1960 - wird erfolgreich gegen Poliomyelitis geimpft, was der Rückgang und die anstehende Zertifizierung der Eliminierung der Erkrankung beweisen. Bereits zwischen 1961 und 1965 hat sich die Zahl der jährlichen Neuerkrankungen um 99\% reduziert, d. h. von 4.670 auf weniger als 50 Fälle. 1986 und 1990 traten in Deutschland die letzten beiden Erkrankungsfäl- 


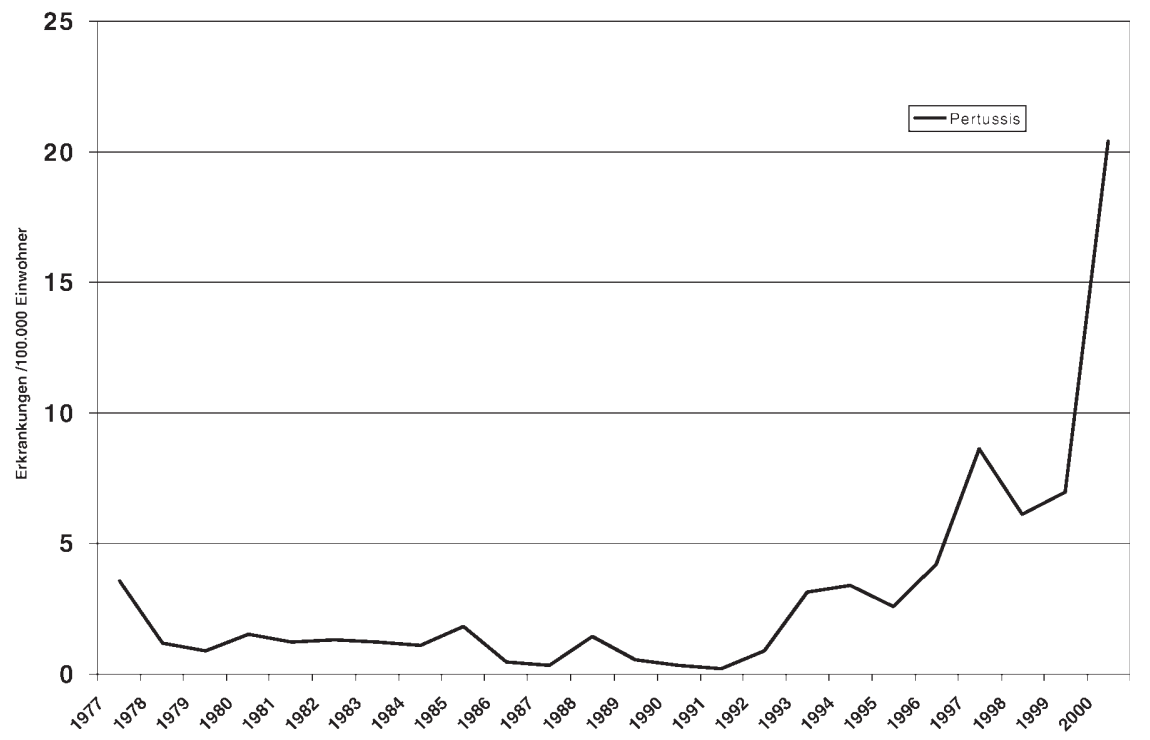

Abb.5 $\triangle$ Pertussisinzidenz in der DDR und in den neuen Bundesländern von 1977-2000

ten Säugling in Finnland [8] unterstreichen, wie wichtig diese Impfung trotz niedriger Inzidenz in Deutschland ist.

\section{Tetanus}

In Deutschland treten im Mittel weniger als 15 Tetanusfälle jährlich auf. 1999 und im Jahr 2000 wurden jeweils acht Fälle erfasst. Die Erkrankung betrifft praktisch nur noch ältere, nicht ausreichend immunisierte Personen. Da der Tetanus gemäß IfSG nicht mehr meldepflichtig ist, wird in Zukunft die Hospitalstatistik die einzige Datenquelle für die weitere

Überwachung der Erkrankungsfälle sein.

\section{Hepatitis B}

Im Jahr 1999 wurden 4.565 Erkrankungen an Hepatitis B gemeldet. Dies stellt einen Rückgang von $12 \%$ gegenüber 1998 ( 5.185 Fälle) dar. Im Jahr 2000 wurden 4.542 Erkrankungen gemeldet und 4.208 Fälle im Jahr 2001. Die Gesamterkrankungszahlen haben in den letzten Jahren also nicht wesentlich abgenommen. 1998 wurde von einer Inzidenz von 6,3 Hepatitis-B-Erkrankungen pro

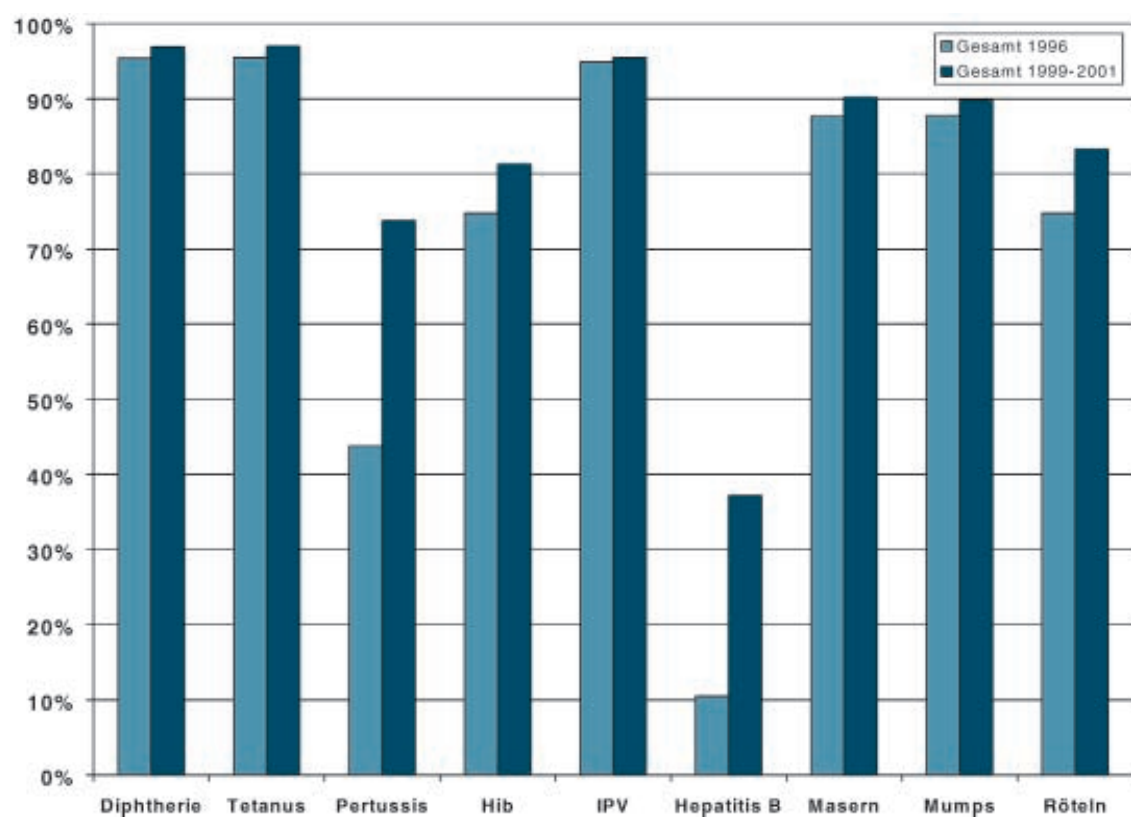

Abb.6 $\triangle$ Durchimpfungsrate bei Schuleintritt im Jahr 1996 im Vergleich zu den Jahren 1999-2001 in \% der vorgelegten Impfausweise
100.000 Einwohner ausgegangen (ABL: 7,3/100.00, NBL: 1,7/100.000). Im Jahr 2000 ist diese auf 5,5/100.000 gesunken (ABL: 6,3/100.000; NBL 1,7/100.000). Nach Ergebnissen des Bundes-Gesundheitssurveys von 1998 hatten $7 \%$ der Bevölkerung Kontakt zum Hepatitis-B-Virus (ABL: $7,7 \%$, NBL: 4,3\%), und Träger des Virus sind o,6\% (ABL: o,6\%,, NBL: $0,4 \%)$. Die relativ niedrigen Erkrankungsraten in den neuen Bundesländern sind vermutlich durch die geringeren Anteile von Risikogruppen bedingt, wie z. B. Drogensüchtige sowie Ausländern aus Hochendemiegebieten.

Seit 1995 empfiehlt die STIKO die Impfung gegen Hepatitis B für alle Säuglinge und - soweit nicht bereits erfolgt das Nachholen der Impfung bis zum vollendeten 18. Lebensjahr. Veränderungen in den Erkrankungshäufigkeiten als Folge dieser Maßnahme sind bei dem insgesamt geringen Anteil der Kinder an der Gesamterkrankungshäufigkeit erst in den nächsten Jahren zu erwarten. Der Rückgang der Erkrankungen bei Kindern unter 15 Jahren belegt jedoch bereits jetzt die Wirksamkeit der Impfung (Inzidenz in der Altersgruppe fünf bis 14 Jahre: 1995:4,4/100.000, 2000: 2,5/100.00; Inzidenz in der Altersgruppe ein bis vier Jahre:1995: 4,2/100.000, 2000: 1,2/100.000). Zur effektiven Eindämmung der Hepatitis B ist zusätzlich zur Umsetzung der generellen Impfempfehlung für alle Kinder bis zum vollendeten 18. Lebensjahr die Umsetzung der Hepatitis-B-Impfung in Risikopopulationen zu fördern.

\section{Hämophilus influenzae Typ b}

Seit 1990 empfiehlt die STIKO die Impfung gegen Hämophilus influenzae Typ b (Hib). Der Rückgang der Erkrankungszahlen belegt die Wirksamkeit und Effektivität dieser Impfung. Wurden 1991 in den neuen Bundesländern und Berlin noch 98 Meningitiserkrankungen durch Hämophilus influenzae registriert, so sind 2000 nur noch sechs Erkrankungen gemeldet worden [9]. Auch im ESPEDMeldesystem werden invasive Infektionen durch Hämophilus influenzae Typ b mit abnehmendem Trend erfasst.

Im Jahr 2000 wurden im ESPEDMeldesystem insgesamt 63 Fälle einer invasiven Hämophilus-influenzae-Infektion bei Kindern unter zehn Jahren erfasst [10]. Unter den 50 typisierten Fäl- 
len befanden sich 23 Fälle mit einer Hämophilus-influenza-Typ-b-Infektion. Hiervon konnten wiederum in 19 Fällen Daten zum Impfstatus erhoben werden. Es zeigte sich, dass zwölf Kinder überhaupt nicht und vier Kinder nicht zeitgerecht geimpft waren. Nur drei Kinder waren zum Zeitpunkt der Erkrankung regelgerecht geimpft. Für ein Kind bestand Unklarheit über eine zugrundeliegende Grunderkrankung, für zwei weitere Kinder wurde diese ausgeschlossen. In diesem Zusammenhang wird die Notwendigkeit der zeitgerechten Impfung nochmals deutlich.

\section{Erfassungssysteme zu Durchimpfungsraten}

Die Erfassung von Durchimpfungsraten ist notwendiger Bestandteil der Planung und der Evaluation von Impfprogrammen und damit Voraussetzung für eine effektive Bekämpfung von impfpräventablen Erkrankungen. Es werden national und international unterschiedliche Methoden der Impferfassung von unterschiedlichen Datenhaltern und Nutzern (z. B. passive und aktive Surveillance, repräsentative Studien oder Register, Sentinels, seroepidemiologische Studien, Krankenkassendaten, Abrechnungsdaten der Kassenärztlichen Vereinigungen, Verkaufsdaten) mit unterschiedlichen Zielen angewandt. Vollständige Erfassungen sind nur über populationsbezogene Impfregister möglich, die jedoch einen erheblichen organisatorischen und finanziellen Aufwand erfordern und Probleme der Datensicherheit und nutzung aufweisen können. Bei Implementation und sachgerechter Nutzung sind populationsgerechte Register aber eine geeignete Methode um Impfraten zu erhöhen [11, 12, 13]. In Deutschland bestehen derzeit in zwei Bundesländern gesetzliche Grundlagen und Bemühungen, eine bevölkerungsbezogene Impferfassung einzuführen.

Weltweit gelten die Durchimpfungsraten, die bis zum Ende des zweiten Lebensjahres erreicht werden, als Qualitätsindikator für die Umsetzung von Impfprogrammen für Säuglinge und Kinder. Sie werden von der WHO und EU herangezogen, um die Qualität der Impfprogramme der Länder untereinander zu vergleichen. Für Deutschland liegen aktuell keine diesbezüglichen Daten vor. Ergebnisse aus einer re- präsentativen Telefonbefragung [14] und einer Erhebung in einer Abteilung für Kinder- und Jugendmedizin [15] lassen gravierende Mängel im altersgerechten Impfschutz erkennen und zeigen, dass gerade zur Dokumentation des altersgerechten und zeitgerechten Impfstatus dringend weitere valide und belastbare Daten benötigt werden. Entsprechende Daten werden sich zum Teil aus dem Kinder- und Jugendsurvey sowie aus einem Forschungsprojekt des Berufsverbandes der Kinderärzte zum Impfverhalten niedergelassener Kinderärzte, das in Zusammenarbeit mit dem RKI durchgeführt wird, gewinnen lassen. Für eine regionale Handlungssteuerung und die Implementation von differenzierten Maßnahmen zur Steigerung der Durchimpfungsrate bis zum Ende des zweiten Lebensjahres sind jedoch diese Daten noch nicht optimal.

Eine gute Basis für die Darstellung der Durchimpfungsraten zu einem bestimmten Zeitpunkt bieten die seit 2001 im $\$ 34$ Abs. 11 IfSG verbindlich geregelten Erhebungen des Impfstatus bei Schuleintritt [1] (s.u.). Differenzierte Analysen zur Vollständigkeit, zur zeitgerechten Durchführung der Impfung und zu Risikofaktoren für das Unterlassen einer Impfung auf Basis dieser Erhebungen sind derzeit aber noch nicht möglich. Aufgrund von Kindergarten- und Schuluntersuchungen liegen aber in einigen Bundesländern (z. B. in SchleswigHolstein, Brandenburg u.a.) bereits Querschnittsergebnisse zu den Durchimpfungsraten zu anderen Zeitpunkten als dem Schuleintritt vor. Sie belegen eindrucksvoll, dass sich aus der Erhebung der Durchimpfungsraten mögliche und notwendige Interventionsmaßnahmen ableiten lassen [16].

Daten über den Durchimpfungsgrad der erwachsenen Bevölkerung stehen sowohl aus dem 1998 durchgeführten Bundes-Gesundheitssurvey [17] als auch aus der Analyse speziell untersuchter Bevölkerungsgruppen (z. B. Beschäftigte im Gesundheitswesen, Reisende) sowie aus wenigen seroepidemiologischen Erhebungen (z. B. [18]) und repräsentativen Befragungen zur Verfügung. Eine bevölkerungsbezogene Querschnittsuntersuchung $\mathrm{zu}$ einem definierten Zeitpunkt (wie bei den Kindern bei Schuleintritt) oder interventionsorientierte bevölkerungsbezogene Ansätze fehlen aber zurzeit völlig. Um zielgerich- tet Interventionsmaßnahmen zur Verbesserung der Durchimpfungsraten in Deutschland $\mathrm{zu}$ implementieren, sind deshalb weitere Datenquellen zu erschließen. Fortschritte sind in diesem Zusammenhang von den im Rahmen der Umsetzung des $\$ 20$ Abs. 4 IfSG in Zusammenarbeit mit den Krankenkassen und Kassenärztlichen Vereinigungen diskutierten zukünftigen Vorgehensweisen $\mathrm{zu}$ erwarten. Insbesondere könnten die Daten der Krankenkassen und Kassenärztlichen Vereinigungen wichtige Informationen über den $\mathrm{Zu}$ sammenhanges zwischen Impfung und Gesundheitsstatus liefern.

\section{Durchimpfungsraten in Deutschland}

Tabelle 4 zeigt auf Basis der Meldungen von 14 Bundesländern die Durchimpfungsraten bei Schuleintritt für die Jahre von 1999 bis 2001 .

Abbildung 6 zeigt den Anstieg der Durchimpfungsraten von Kindern bei Schuleintritt in den Jahren 1999-2000 gegenüber dem Jahr 1996.

Der Impfschutz der Vorschulkinder gegen Diphtherie $(96,9 \%)$, Tetanus (97\%) und Poliomyelitis (95,5\%) ist gegnüber 1996 weiter angestiegen. Der Impfschutz gegen Masern (90,2\%), Mumps $(89,8 \%)$ und Röteln $(83,3 \%)$ ist trotz steigender Tendenz noch nicht zufriedenstellend und für eine Eliminierung der Masern unzureichend. Ursache hierfür ist insbesondere die völlig unzureichende Durchführung $(14,4 \%)$ der zweiten MMR-Impfung (MasernMumps-Röteln-Impfung). Die diesbezügliche Durchimpfung ist weiter $\mathrm{zu}$ verbessern. Gegenüber den Ergebnissen von Schuleingangsuntersuchungen aus dem Jahr 1996 haben sich die Impfraten gegen Haemophilus influenzae Typ b (von $54,7 \%$ auf $81,3 \%$ ) und auch gegen Pertussis (von $34,5 \%$ auf $73,8 \%$ ) zwar deutlich erhöht, es sind aber auch hier noch große Impflücken zu erkennen. Für Pertussis ist die Durchimpfungsrate der eingeschulten Kinder in den alten Bundesländern mit $71,9 \%$ noch immer sehr unbefriedigend (neue Bundesländer $88,6 \%$ ), auch wenn hier in den letzten Jahren ein kontinuierlicher Anstieg zu beobachten ist. Die Umsetzung der STIKO-Empfehlung 2000, die aufgrund der epidemiologischen Entwicklung bei Pertussiserkrankungen und der be- 
kannten fehlenden lebenslangen Immunität ein Nachholen der Grundimmunisierung bzw. eine Wiederauffrischung für Jugendliche vorsieht, ist deshalb für die Verhinderung der Weiterverbreitung der Pertussis sowie die Minderung der Erkrankungszahlen von besonderer Bedeutung.

Die Durchimpfungen gegen Hepatitis B hat ebenfalls zugenommen (1999/ 2001: ABL: 39\%, NBL: 27,0\%, gesamt: 37,2\%). 1996 waren die Schulanfänger nur zu $8 \%$ gegen Hepatitis B geimpft. Die Impfraten sind für einen völligen Schutz dieser Altersgruppe jedoch noch immer völlig unzureichend, vor allem weil Nachholimpfungen im Jugendalter nicht oder nur unzureichend genutzt werden. Die in der Vergangenheit zum Teil restriktiv gehandhabte Finanzierung dieser Impfung durch die Krankenkassen hat zur unzureichenden Durchimpfung in dieser Altersgruppe beigetragen. Zukünftig ist allerdings aufgrund des seit einiger Zeit zur Verfügung stehenden Kombinationsimpfstoffes mit einem deutlichen Anstieg der Durchimpfungsraten im Kleinkindalter zu rechnen. Auch finanzielle und strukturelle Vereinbarungen zwischen Krankenkassen, kassenärztlichen Vereinigungen und Ländern dürften hier unterstützend wirken.

Daten, die zum Zeitpunkt des Schuleintrittes erhoben werden, geben keinen Hinweis darauf, ob die von der STIKO empfohlenen Impfungen altersgerecht und zeitgerecht durchgeführt werden. In der repräsentativen Studie von Laubereau [14] konnte gezeigt werden, dass nur 59\% (95\% Konfidenzintervall: 54\%-64\%) der Kinder mit 19 Monaten eine vollständige Grundimmunisierung erhalten hatten und mit sieben Monaten erst 50\% der Kinder altersgerecht grundimmunisiert waren. Diese Verzögerung gilt auch für die Boostergabe im zweiten Lebensjahr. Nur 50\% der 19 Monate alten Kinder besaßen einen vollständigen Impfschutz (Grundimmunisierung und Boosterung). Aus diesen Erhebungen lässt sich schließen, dass ein großer Teil der Impfungen $\mathrm{zu}$ spät und in nicht zeitgerechten Abständen verabreicht wird. Da gerade der Immunschutz im ersten Lebensjahr von Bedeutung ist, sind in Zukunft vermehrt Anstrengungen zu unternehmen, Impfungen so früh und vollständig wie möglich zu verabreichen. Impfregister und automatisierte Recallsysteme könnten hierzu einen wertvollen Beitrag leisten [12].

Daten aus den Kindergartenuntersuchungen [16] bestätigen die mangelnde Vervollständigung von Impfungen, weisen jedoch zusätzlich auf ein weiteres Problem hin: das Nachholen von Impfungen entsprechend den STIKOEmpfehlungen. In Schleswig-Holstein sind z. B. 78,6\% der 2,5- bis dreijährigen Kinder gegen Hepatitis B geimpft, im Alter von 5,5 bis sechs Jahren sind es aber nur $58,7 \%$. Obwohl die STIKO das Nachholen einer neu eingeführten Impfung (Hepatitis B 1995) empfiehlt, wird die Empfehlung trotz bestehender Vorsorgeprogramme (U1-U9, J1) nur unzureichend umgesetzt. Hier mögen Fragen der Impfstoffverfügbarkeit und der Kostenübernahme eine große Rolle spielen. Die bekannte fehlende Nutzung von Arztkontakten zur Vervollständigung und Aktualisierung des Impfstatus ist vermutlich eine weitere Ursache für das unzureichende Nachholen empfohlener Impfungen.

Werden Impfungen bei Kindern noch vergleichsweise gut akzeptiert, weisen Erwachsene große Impflücken auf, und die Datenlage zu Durchimpfungsraten im Erwachsenenalter ist spärlich. Daten des Bundes-Gesundheitssurveys von 1998 zeigen, dass nur $63 \%$ der befragten Erwachsenen in den letzten zehn Jahren eine Auffrischimpfung gegen Tetanus erhielten, wobei sich die Impfraten mit abnehmendem Lebensalter erhöhten ( $82 \%$ der 18 - bis 19 Jährigen). Fernreisende [17] und Bewohner der neuen Bundesländer weisen grundsätzlich einen besseren Durchimpfungsgrad auf als Bewohner der alten Bundesländer und Nichtreisende. Zudem verdeutlichen Daten aus einer europaweiten Seroprävalenzstudie, die auf Basis der Analyse von Restseren aus den Jahren 1995-1996 erhoben wurden, dass in Deutschland bei Erwachsenen Impflücken für Diphtherie und Pertussis bestehen [18]. Auch werden Indikationsimpfungen sowohl für besondere Risikogruppen (Pneumokokkenimpfung und Influenzaimpfung für Senioren) als auch für Beschäftigte (z.B. Influenzaimpfung für Beschäftigte im Gesundheitswesen) sowie bei Vorliegen gesundheitlicher Beeinträchtigungen (z.B. Influenzaimpfung bei Diabetes mellitus) völlig unzureichend umgesetzt [18].

\section{Gründe für niedrige Durch- impfungsraten und Strategien zur Verbesserung der Durchimpfungsraten}

Wegen der Freiwilligkeit von Impfungen ist ihre Akzeptanz von erheblicher Bedeutung für die effektive Umsetzung von Impfstrategien. Das Ausmaß dieser Akzeptanz wird von vielen verschiedenen Faktoren beeinflusst. Aufgrund erfolgreicher Impfprogramme treten impfpräventable Erkrankungen heute selten auf. Diese Tatsache bedingt wiederum ein fehlendes Bewusstsein über die möglichen Komplikationen der Erkrankungen und mindert die Akzeptanz von Impfungen. Hinzu kommt ein unzureichendes Wissen über die Wirkungsweise, den Nutzen und die Wirksamkeit der Impfung. Seltene Nebenwirkungen rücken deshalb in den Vordergrund der Wahrnehmung der Bevölkerung und führen dazu, dass notwendige und effektive Impfmaßnahmen abgelehnt werden. Falsch verstandene Kontraindikationen tragen ebenfalls zur geringen Wertschätzung bei. Es gilt also die Informationsdefizite der Bevölkerung, des Fachpublikums aber auch der Gesundheitspolitik zu verringern. Zur Umsetzung des Impfgedankens ist die Unterstützung sämtlicher Akteure im Gesundheitswesen sowie seine breite Verankerung im gesundheitspolitischen Prozess unerlässlich.

Voraussetzung für eine effektive Aufklärung und die Konzeption effektiver Impfprogramme ist die Verbesserung der Datenlage zur Häufigkeit, zur Verteilung und zu Risikofaktoren impfpräventabler Erkrankungen sowie zu Durchimpfungsraten und zur Wirksamkeit von Impfstrategien. Erforderlich ist zudem die Verfügbarkeit umfangreicher und valider Daten zu Impfnebenwirkungen und Komplikationen, da Transparenz und Aufklärung die Impfakzeptanz erhöht. Dieser Informations- und Aufklärungsprozess, der auch im IfSG $\left(\$ \$_{3}\right.$, 20 Abs.1) betont wird, muss durch gesundheitspolitische Zielsetzungen mit Betonung des Präventivgedankens ergänzt werden.

Zusätzlich zu den oben genannten Informations- und Aufklärungsdefiziten verhindern Defizite in Strukturen und Prozessen im Gesundheitswesen das Erreichen von höheren Durchimpfungsraten. Hier ist zum einen die immer noch 
unzureichende Nutzung des Arzt-Patienten-Kontaktes zur Aktualisierung des Impfstatus zu nennen. Diese fehlende Aktualisierung ist nicht alleine durch die Nachlässigkeit des Arztes sondern auch durch unzureichende bzw. uneinheitliche Honorierung von Impfungen bedingt. Zur Stärkung des primär präventiven Gedankens sind daher neue Impulse auch auf der Ebene der Kostenerstattung aus den anstehenden Verhandlungen mit den Gremien der Selbstverwaltungen und den Krankenkassen unerlässlich. Ebenfalls erschwert die mangelnde Vernetzung der einzelnen Akteure und Handlungsebenen im Impfwesen eine effektive Umsetzung von Impfprogrammen. Entsprechend sind in vielen Bundesländern Impfvereinbarungen getroffen worden und Projekte entstanden, die versuchen, die Verzahnung von niedergelassenen Ärzten, dem öffentlichen Gesundheitsdienst (ÖGD), Krankenkassen und Gesundheitsverwaltungen zu verbessern [16]. Die Ergebnisse dieser Projekte machen deutlich, dass durch Koordination und Kooperation Hindernisse in der Umsetzung von Impfprogrammen vermeidbar sind und Durchimpfungsraten gesteigert werden können. Inwieweit hier auch strukturelle Veränderungen z.B.im Sinne der Schaffung einer multidisziplinären Institution zur Förderung des Impfgedankens in Deutschland erfolgreich und notwendig sein könnten, ist Gegenstand der aktuellen Diskussion.

Unterschiedliche Zugangswege und Inanspruchnahmebarrieren besonders von Risikogruppen sowie weitere soziale Faktoren (Nationalität, Schichtzugehörigkeit, Bildungstand, Region, u.a.) spielen bei der fehlenden Umsetzung von empfohlenen Impfungen ebenfalls eine große Rolle (z.B. $[15,19]$ und sollten in Zukunft bei der Ausgestaltung von Impfprogrammen vermehrt berücksichtigt werden.

Handlungsempfehlungen und Maßnahmen zur Erhöhung von Durchimpfungsraten sind vielfältig und berücksichtigen unterschiedliche Handlungsebenen, Zielgruppen und Interventionen. Hier können unterschiedliche Interventionsebenen unterschieden werden: Es können die Nachfrage nach Impfungen erhöht, der Zugang erleichtert und gesetzliche Regelungen vereinfacht bzw. verbessert sowie Nutzer oder Anbieter orientiert strukturiert werden [12, 2o]. Effektiv sind Programme, wenn sie mehrere Handlungsebenen und Interventionsrichtungen miteinander verbinden. Als Maßnahmen mit nachgewiesener Evidenz zur Steigerung der Durchimpfungsraten [12] gelten automatische Erinnerungs- oder Recallsysteme, Impfregister, Aufklärungsmaßnahmen für Bevölkerung und Fachöffentlichkeit, Kostenerstattung, Abbau von Zugangsbarrieren und Qualitätssicherungsmaßnahmen. Das RKI hat 1998 ein Zehnpunkteprogramm zur Erhöhung der Impfbereitschaft und zur Steigerung der Durchimpfungsraten in Deutschland formuliert sowie aktuelle und prioritäre Handlungsfelder zur Verbesserung der Impfsituation aufgezeigt [21]. In einigen Bereichen wurden bereits beeindruckende Teilerfolge erzielt, insbesondere bietet das IfSG wichtige Neuerungen sowohl für die Verbesserung der Datenlage als auch für die Rolle des ÖGD und die Stärkung des Informations- und Aufklärungsgedanken. Defizite sind jedoch weiterhin insbesondere in der Einrichtung von Erinnerungssystemen oder Impfregistern, in der Kostenregelung, in der Aus- und Weiterbildung sowie in der Nutzung bestehender Strukturen im Gesundheitswesen und deren Vernetzung festzustellen. Hier gilt es, für die Zukunft Ziele zu entwickeln und Maßnahmen zu implementieren, die diese noch unzureichend umgesetzten Punkte verstärkt berücksichtigen.

\section{Literatur}

1. Robert Koch-Institut (2000) Die Bedeutung des Infektionsschutzgesetzes für die Impfprävention übertragbarer Krankheiten. Bundesgesundheits Gesundheitsforsch Gesundheitsschutz 43:882-886

2. Robert Koch-Institut (2001) Masern-Surveillance mit zwei Erfassungssystemen. Epid Bull 38:295-298

3. Robert Koch-Institut (2001) Sentinel Surveillance der Arbeitsgemeinschaft Masern. Weitere Ergebnisse. Epid Bull 14:98-100

4. Robert Koch-Institut (2000) Sentinel-Surveillance der Arbeitsgemeinschaft Masern. Ergebnisse und Erfahrungen. Epid Bull 49:387-390

5. Robert Koch-Institut (2001) Zu den Erkrankungen im Jahr $2000 \mathrm{im}$ Land Brandenburg. Epid Bull 34:260-261

6. Juretzko P, Fabian-Marx T, Haastert B, Giani G, Kries v. R, Wirsing v. König CH (2001) Pertussis in Germany: regional differences in management and vaccination status of hospitalized cases. Epidemiol Infect 127:63-71
7. Communicable Disease Surveillance Center (2002) Toxigenic corynebacterium diphtheriae var miitis isolated from a child from Salford. Comm Dis Reg CDR Weekly 12:4

8. (2000) Fatal case of diphteria in an unvaccinated infant, Finnland 2001. Eurosurveillance Weekly 6

9. Robert Koch-Institut (2001) Infektionen des Zentralnervensystems. Epid Bull 48:366-368

10. Creutzig U, Forster J, Giani G et al (2001) ESPEDJahresbericht 2000. Monatsschr Kinderheilkd 149:1268-1277

11. LeBaron C, Chaney M, Baughman A, Dini E, Mases E, Dietz V, Bernier R (1997) Impact of measurement and feedback on vaccination coverage in public clinics 1988-1994. JAME 227:631-635

12. Briss P, Rodewald L, Hinmann A et al. (2000) Review of evidence regarding interventions to improve vaccination coverage in childen, adolescents, and adults. Am J Prev Med 18:(19)97-140

13. Stokley S, Rodewald L.Mases E (2001) The impact of record scattering on the measurement of immunization coverage. Pediatrics 107:91-96

14. Laubereau B, Hermann M. Weil J, Schmitt HJ, Kries R (2001) Durchimpfungsraten bei Kindern in Deutschland 1999. Monatsschr Kinderheilkd 149:367-372

15. Kunze W, Kasek K, Hausen D (2000) Erfassung von Impfstatus und soziologischen Daten bei Patienten im Kindes- und Jugendalter. Immunologie Impfen 3:99-107

16. Robert Koch-Institut (2002) Erfassung des Impfstatus bei Aufnahme in den Kindergarten. Epid Bull 4:25-28

17. Altmann A, Breuer T, Rasch G (1999) Impfstatus und Erkrankungen bei Fernreisenden. Gesundheitswesen 61 [Suppl 2]:106-109

18. Robert Koch-Institut (1999) Populationsimmunität bei Diphtherie und Pertussis. Epid Bull 1:1-4

19. Ellsäßer G (1998) Gesundheit von Schulanfängern: Auswirkung sozialer Benachteiligung am Beispiel der Brandenburger Schulanfängeruntersuchungen 1994 und 1995. Gesundheitswesen 60:632-637

20. Shefer A, Briss P, Rodewald L et al. (1999) Improving immunization coverage rates: an evidencebased review of the literature. Epidemiol Rev 21:96-142

21. Robert Koch-Institut (1999) Zehnpunkteprogramm zur Erhöhung der Impfbereitschaft und zur Steigerung der Durchimpfungsraten in Deutschland. Bundesgesundheits Gesundheitsforsch Gesundheitsschutz 42:290-292 